\begin{tabular}{|c|l|}
\hline Title & MA IC-2, a latitudinal model for the Martian surface temperature, atmospheric water transport and surface glaciation \\
\hline Author(s) & Greve, Ralf; Grieger, Björn; Stenzel, Oliver J. \\
\hline Citation & $\begin{array}{l}\text { Planetary and Space Science, 58(6), 931-940 } \\
\text { https://doi.org/40.1016/.pss.2010.03.002 }\end{array}$ \\
\hline Issue Date & 2010-05 \\
\hline Doc URL & http://hdl.handle.net/2115/43084 \\
\hline Type & article(author version) \\
\hline File Information & Greve_etal_2010_PSS.pdf \\
\hline
\end{tabular}

Instructions for use 


\title{
MAIC-2, a latitudinal model for the Martian surface temperature, atmospheric water transport and surface glaciation
}

\author{
RALF GREVE* \\ Institute of Low Temperature Science, Hokkaido University, \\ Kita-19, Nishi-8, Kita-ku, Sapporo 060-0819, Japan \\ BJÖRN GRIEGER \\ European Space Astronomy Centre, P.O. Box - Apdo. de Correos 78, \\ 28691 Villanueva de la Cañada, Madrid, Spain \\ Oliver J. Stenzel \\ Max Planck Institute for Solar System Research, \\ Max-Planck-Straße 2, 37191 Katlenburg-Lindau, Germany
}

\begin{abstract}
The Mars Atmosphere-Ice Coupler MAIC-2 is a simple, latitudinal model, which consists of a set of parameterisations for the surface temperature, the atmospheric water transport and the surface mass balance (condensation minus evaporation) of water ice. It is driven directly by the orbital parameters obliquity, eccentricity and solar longitude $\left(L_{\mathrm{s}}\right)$ of perihelion. Surface temperature is described by the Local Insolation Temperature (LIT) scheme, which uses a daily and latitude-dependent radiation balance. The evaporation rate of water is calculated by an expression for free convection, driven by density differences between water vapor and ambient air, the condensation rate follows from the assumption that any water vapour which exceeds the local saturation pressure condenses instantly, and atmospheric transport of water vapour is approximated by instantaneous mixing. Glacial flow of ice deposits is neglected. Simulations with constant orbital parameters show that low obliquities favour deposition of ice in high latitudes and vice versa. A transient scenario driven by a computed history of orbital parameters over the last 10 million years produces essentially monotonically growing polar ice deposits during the most recent 4 million years, and a very good agreement with the observed present-day polar layered deposits. The thick polar deposits sometimes continue in thin ice deposits which extend far into the mid latitudes, which confirms the idea of "ice ages" at high obliquity.
\end{abstract}

*E-mail: greve@lowtem.hokudai.ac.jp 


\section{Introduction}

On time scales of $10^{5}-10^{6}$ years, Mars has experienced large periodic changes of the orbital elements obliquity, eccentricity and equinox precession. These changes have an impact on the Martian climate. The obliquity determines the strength of the seasons and the latitudinal distribution of mean solar insolation. The eccentricity determines the magnitude of the asymmetry of insolation with season, and the equinox precession determines the timing of the asymmetry of solar insolation with season. On Earth, the so-called Milankovitch cycles of much weaker orbital changes with periods of 20, 40 and 100 ka are considered driving forces for climate variations like the glacial/interglacial cycles. It can, therefore, be expected that the main Martian $\pm 10^{\circ}$ obliquity cycles with periods of 125 ka and $1.3 \mathrm{Ma}$ and the secular shift from high to low average obliquities at 4-5 Ma ago (Laskar et al. 2004, shown in Fig. 1) have significant impacts on the climate and the polar layered deposits (PLDs) due to large insolation changes in the polar regions.

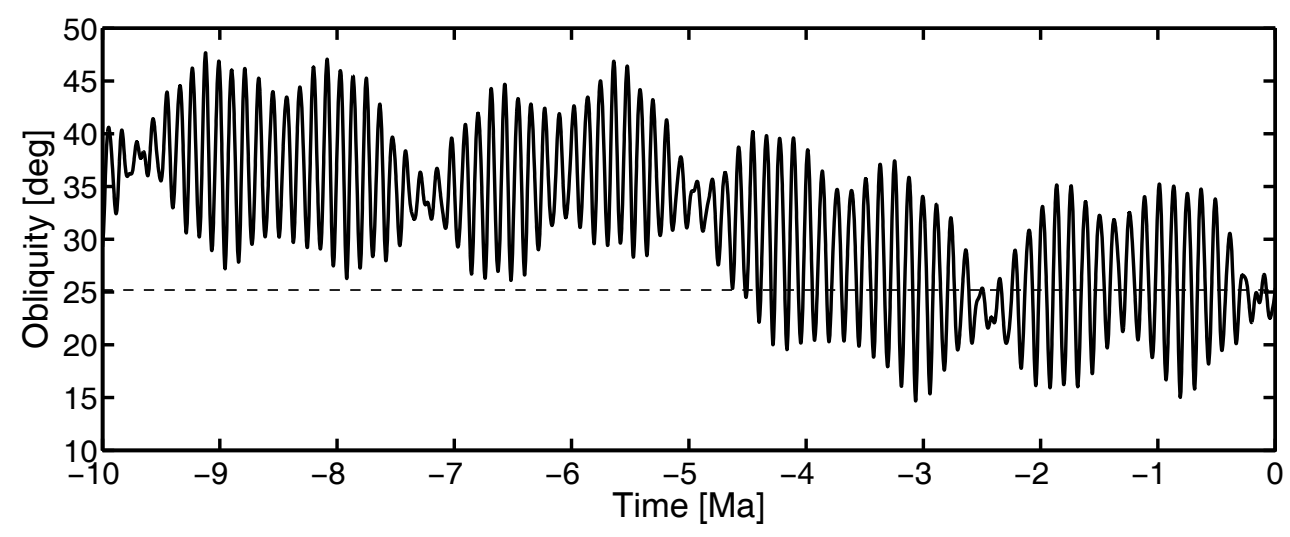

Figure 1: Martian obliquity for the last 10 Ma (Laskar et al. 2004).

This idea is supported by the presence of light-dark layers in the PLDs, which are exposed in the surface troughs and close to the margins, and which are actually the reason for the term "polar layered deposits". These layers indicate a strongly varying dust content of the ice due to varying climatic conditions in the past. During periods of high obliquities, insolation in the polar regions is large, which entails higher sublimation rates of superficial ice of the PLDs and probably of permafrost in the ground. This may lead to the formation of a thicker and dustier atmosphere, so that dust accumulates on the PLDs. By contrast, during periods of low obliquities, the atmosphere is thin and dust deposition is low, so that clean ice forms at the surface of the PLDs. Along this line of reasoning, Head et al. (2003) presented evidence for past glaciation in the mid-latitudes and suggested that Mars experienced "ice ages" during periods of high obliquity like that from about 2.1 to $0.4 \mathrm{Ma}$ ago (with obliquity maxima of $\approx 35^{\circ}$ ). These ice ages were supposedly characterised by warmer polar climates, enhanced mass loss of the PLDs due to sublimation and the formation of metres-thick ice deposits equatorward to approximately $30^{\circ} \mathrm{N} / \mathrm{S}$.

In a number of studies, General Circulation Models (GCMs) have been applied to the Martian atmosphere (e.g., Pollack et al. 1990, Read et al. 1997, Forget et al. 1999, Richardson and Wilson 2002, Haberle et al. 2003, Takahashi et al. 2003). These models, all derivatives of Earth GCMs, solve the equations of fluid dynamics and thermodynamics 
and include e.g. the processes of radiative transfer, cloud formation, regolith-atmosphere water exchange, and advective transport of dust and trace gases. However, they have essentially been designed to simulate the present-day atmosphere in as much detail as possible, and thus are computationally too expensive to permit long-term paleoclimate studies. Segschneider et al. (2005) and Stenzel et al. (2007) adapted an Earth System Model of Intermediate Complexity (EMIC) to Mars. This "Planet Simulator Mars" (PlaSim-Mars, formerly called "Mars Climate Simulator") allows in principle simulations over longer, climatological time scales. So far, only scenarios for present-day conditions and varied obliquity angles have been considered, and the impact on the PLDs has been studied by coupling PlaSim-Mars with the three-dimensional, dynamic/thermodynamic ice-sheet model SICOPOLIS (http://sicopolis.greveweb.net/). In addition to that, simple one-dimensional models have been used to study specific processes that do not require full solution of the dynamic equations. Examples are the radiative transfer model by Gierasch and Goody (1968), the energy balance model by Armstrong et al. (2004), regolith-atmosphere water exchange (Jakosky 1985), and formation of water ice clouds (Michelangeli et al. 1993).

In this study we aim at simulating the surface glaciation of the entire planet with a simple model that depends only on latitude and time. This model, termed the Mars Atmosphere-Ice Coupler Version 2, or MAIC-2 in short, consists of a set of parameterisations for the surface temperature, the atmospheric water transport and the surface mass balance (condensation minus evaporation) of water ice. It is driven directly by the orbital parameters obliquity, eccentricity and solar longitude $\left(L_{\mathrm{s}}\right)$ of perihelion, which were published by Laskar et al. (2004) for the period from 20 million years ago until 10 million years into the future. MAIC-2 is applied to two different kinds of scenarios, namely (i) scenarios with orbital parameters kept constant over time, and (ii) transient scenarios forced by the history of orbital parameters over the last 10 million years. The evolution of surface glaciation is studied for these scenarios under the simplifying assumption of negligible glacial flow, so that changes of local ice thickness are exclusively determined by the local surface mass balance provided by MAIC-2.

\section{Model MAIC-2}

The design of MAIC-2 is illustrated schematically in Fig. 2. All quantities are latitude $(\varphi)$ and time $(t)$ dependent, with the exception of the atmospheric water content. Since instantaneous mixing is assumed, only the (time dependent) global mean water content is modelled. The formulation of the different processes is detailed in the following sections.

\subsection{Timekeeping and orbital position}

We use the timekeeping of PlaSim-Mars (see Sect. 1), in which a Martian year consists of 12 months of 56 days (sols) length. A day has 24 hours of 61.5 minutes length, and a minute is 60 SI seconds long.

In order to compute the relation between solar longitude $L_{\mathrm{s}}$ and time $t$, let us identify the beginning of a Martian year with the northern hemisphere vernal equinox $\left(L_{\mathrm{s}}=0^{\circ}\right)$. 


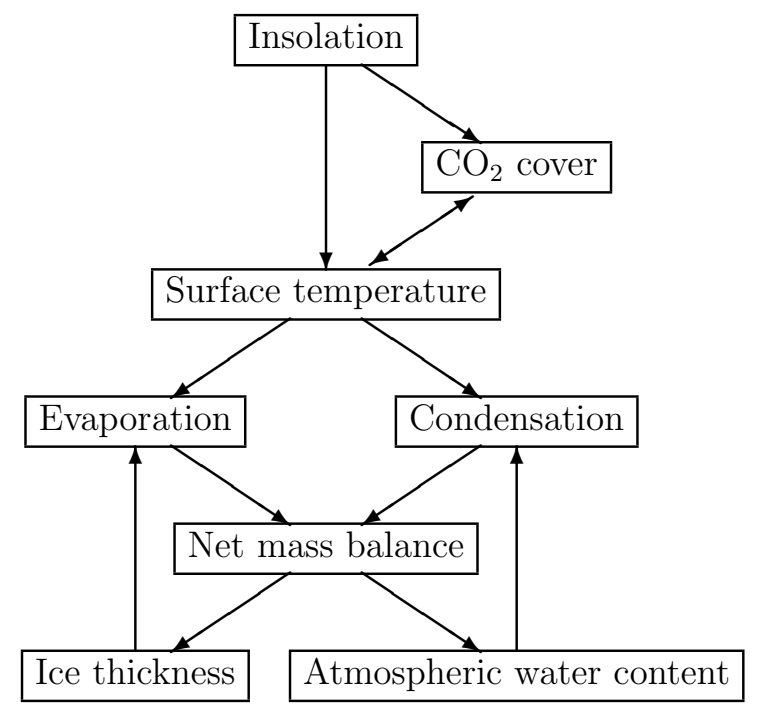

Figure 2: Schematic illustration of the quantities and processes modelled by MAIC-2.

The orbit of Mars around the sun is described by the ellipse

$$
r(\psi)=\frac{p}{1+\varepsilon \cos \psi},
$$

where $r$ is the distance Sun - Mars, $\psi$ the orbital position measured from the perihelion ("true anomaly"), $p$ the semi-latus rectum and $\varepsilon$ the eccentricity. By combining this equation and the conservation of angular momentum $l$,

$$
l=m r^{2} \dot{\psi}
$$

(where $m$ denotes the mass of Mars), we find

$$
\dot{\psi}=\frac{l}{m r^{2}}=\frac{l}{m p^{2}}(1+\varepsilon \cos \psi)^{2}=\Omega(1+\varepsilon \cos \psi)^{2} \quad\left(\Omega:=\frac{l}{m p^{2}}\right) .
$$

Let $L_{\mathrm{s}, \mathrm{p}}$ be the solar longitude of perihelion, then

$$
\psi=L_{\mathrm{s}}-L_{\mathrm{s}, \mathrm{p}},
$$

and since $L_{\mathrm{s}, \mathrm{p}}$ varies only slowly over time, we can rewrite Eq. (3) as

$$
\dot{L}_{\mathrm{s}}=\Omega\left[1+\varepsilon \cos \left(L_{\mathrm{s}}-L_{\mathrm{s}, \mathrm{p}}\right)\right]^{2} .
$$

This equation can be integrated numerically over a full Martian year (from vernal equinox to vernal equinox) for any values of $\varepsilon$ and $L_{\mathrm{s}, \mathrm{p}}$ by a simple Euler-forward scheme. The initial condition is $L_{\mathrm{s}}=0^{\circ}$, and the parameter $\Omega$ is adjusted iteratively such that after one Martian year the orbit is closed $\left(L_{\mathrm{s}}=360^{\circ}\right)$, starting from the initial guess $\Omega_{\text {init }}=$ $2 \pi /(1$ Martian year) [which is the correct value for a circular orbit with $\varepsilon=0$ ].

For present-day conditions $\left(\varepsilon=0.093, L_{\mathrm{s}, \mathrm{p}}=251.0^{\circ}\right)$, the result is shown in Fig. 3. Since the eccentricity is much larger for Mars than for Earth, the relation between $L_{\mathrm{s}}$ and $t$ is significantly different from a linear one. The deviation becomes as large as $21^{\circ}$ $\left(L_{\mathrm{s}}=158.97^{\circ}\right.$ instead of $180^{\circ}$ for day of year 336, leading to a lag of the northern autumnal equinox by 37.7 Martian days). 


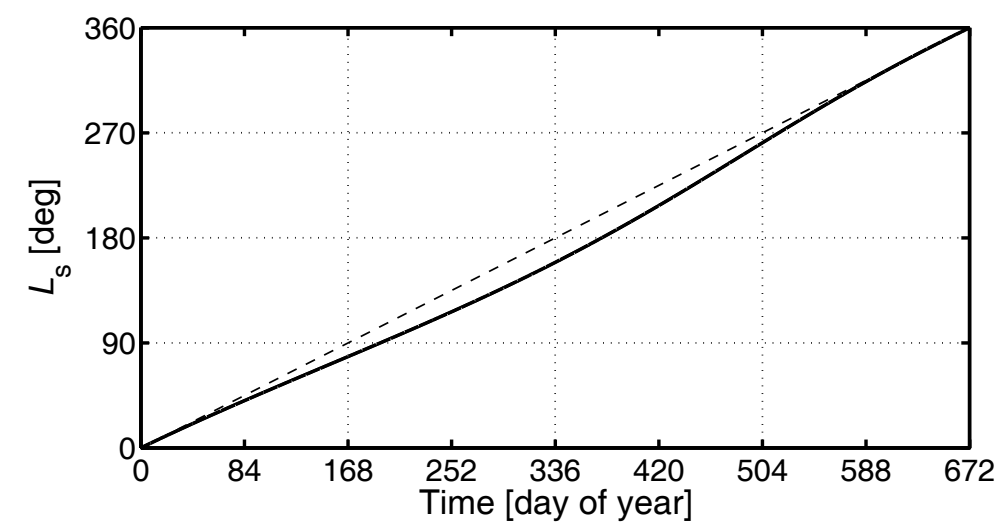

Figure 3: Relation between solar longitude $L_{\mathrm{s}}$ and time $t$ for present-day conditions (solid line). For comparison, the dashed line shows the linear relation for a circular orbit.

\section{$2.2 \quad$ Surface temperature}

In order to derive a parameterisation for the daily mean local surface temperature $T(\varphi, t)$ (depending on latitude $\varphi$ and time $t$ ), we start with the radiation balance

$$
\sigma T^{4}=(1-A) F
$$

where $\sigma$ is the Stefan-Boltzmann constant $\left(\sigma=5.67 \times 10^{-8} \mathrm{~W} \mathrm{~m}^{-2} \mathrm{~K}^{-4}\right), A$ is the surface albedo (globally $A=0.3$ assumed) and $F$ is the local daily mean insolation as a function of the orbital parameters obliquity, eccentricity and solar longitude of perihelion (Laskar et al. 2004). In the absence of seasonal $\mathrm{CO}_{2}$ frost, Eq. (6) provides reasonable results for the surface temperature. However, the equation does not account for the fact that at

$$
T=T_{\text {cond }}=\frac{b}{a-\ln P[\mathrm{hPa}]}
$$

(where $P$ is the surface pressure, $a=23.3494$ and $b=3182.48 \mathrm{~K}$; James et al. 1992) condensation of the atmospheric $\mathrm{CO}_{2}$ (formation of the seasonal ice cap) sets in. The seasonal variation of the surface pressure is neglected, and we use the global annual mean value $P=700 \mathrm{~Pa}$ instead. For this value, Eq. (7) yields a condensation temperature of $T_{\text {cond }}=148.7 \mathrm{~K}$. Since the atmosphere never freezes out completely, this value constitutes the minimum of surface temperatures which can be realised.

In order to find out when the seasonal $\mathrm{CO}_{2}$ ice cap at a given latitude $\varphi$ is present, and therefore $T=T_{\text {cond }}$ holds, the seasonal cap is assumed to exist between the onset of the polar night $\left(t_{\text {dusk }}\right)$ and an unknown time $t$ after the end of the polar night $\left(t_{\text {dawn }}\right)$. During the polar night, condensation takes place, and the amount of formed $\mathrm{CO}_{2}$ frost corresponds to the energy (per area unit)

$$
W_{\text {cond }}=\int_{t_{\text {dusk }}}^{t_{\text {dawn }}} \sigma T_{\text {cond }}^{4} \mathrm{~d} t .
$$

After dawn, the solar insolation causes the $\mathrm{CO}_{2}$ frost to evaporate, which requires the energy

$$
W_{\text {evap }}=\int_{t_{\text {dawn }}}^{t}\left((1-A) F-\sigma T_{\text {cond }}^{4}\right) \mathrm{d} t
$$


The time $t$ at which the $\mathrm{CO}_{2}$ frost has evaporated completely follows from

$$
W_{\text {evap }}=W_{\text {cond }}
$$

The scheme defined by the radiation balance (6), modified by $\mathrm{CO}_{2}$ condensation following Eqs. (7)-(10), is referred to as Local Insolation Temperature scheme (LIT); it was first laid down by B. Grieger (2004; talk at 2nd MATSUP workshop, Darmstadt, Germany). The resulting daily mean surface temperatures over one Martian year for present-day conditions are shown in Fig. 4. They agree well with the data given in the Mars Climate Database (Lewis et al. 1999). The most notable discrepancy is that the LIT scheme overpredicts the summer temperatures at and very close to the poles.
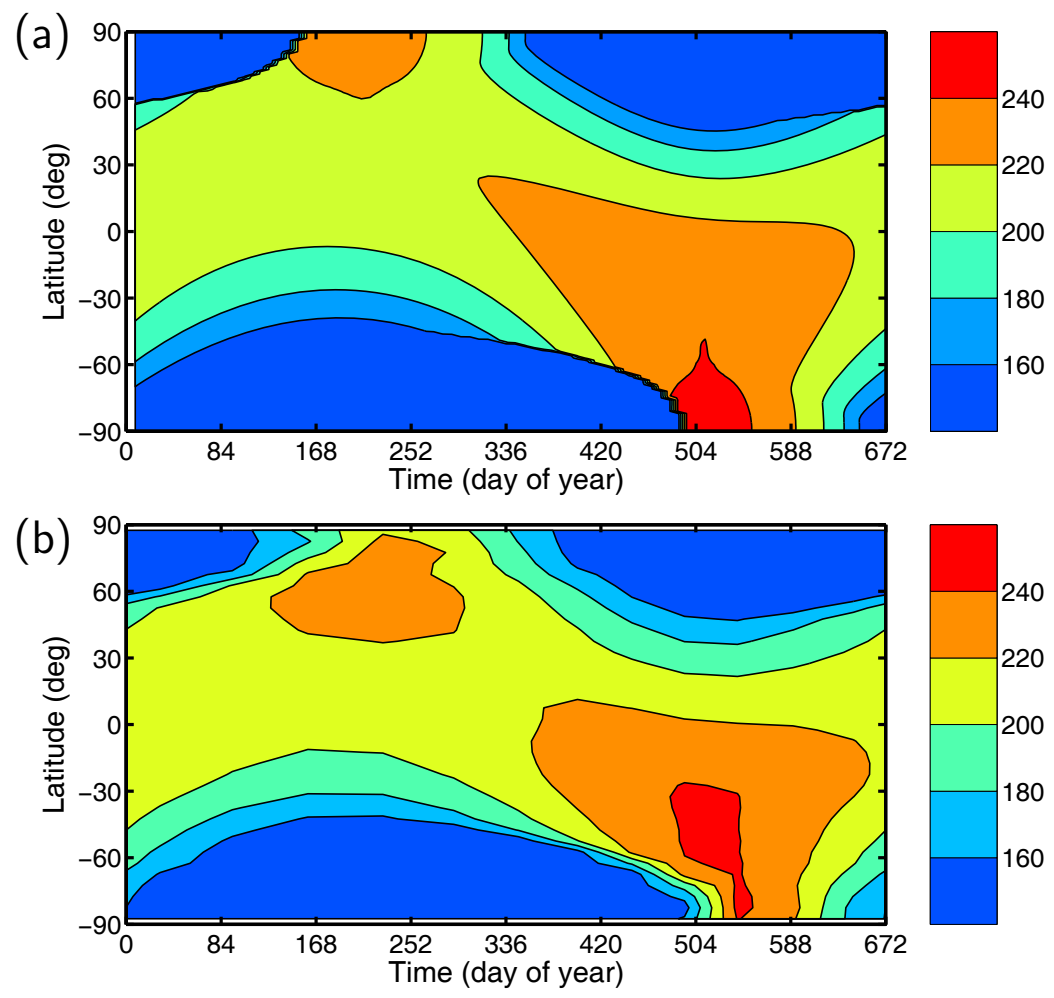

Figure 4: (a) Daily mean surface temperature (in K) of the LIT scheme for present-day conditions. (b) Same, but from the Mars Climate Database (Lewis et al. 1999).

The Mars Atmosphere-Ice Coupler with the LIT scheme and the simple treatment of the surface mass balance described by Greve et al. (2004) and Greve and Mahajan (2005) is referred to as "MAIC-1.5". It was used by these authors to drive simulations of the north polar layered deposits with the ice-sheet model SICOPOLIS.

\subsection{Saturation pressure of water vapour}

The water-vapour saturation pressure $P_{\text {sat }}$ is obtained from the Clausius-Clapeyron relation, which can be integrated only approximately. Different approximations are available; 
we use the Magnus-Teten formula for water vapour over ice (Murray 1967)

$$
P_{\text {sat }}(T)=A \exp \left(\frac{B\left(T-T_{0}\right)}{T-C}\right),
$$

with $A=610.66 \mathrm{~Pa}, B=21.875, C=7.65 \mathrm{~K}$ and $T_{0}=273.16 \mathrm{~K}$, which has also been implemented in the Planet Simulator Mars (Stenzel et al. 2007).

\subsection{Evaporation}

Ingersoll (1970) discussed the water vapour mass flux in the Martian carbon dioxide atmosphere. The evaporation rate $E$ of water from the surface, expressed as a mass flux in $\mathrm{kg} \mathrm{m}^{-2} \mathrm{~s}^{-1}$, is

$$
E=E_{0} \times 0.17 \Delta \eta \rho D\left(\frac{(\Delta \rho / \rho) g}{\nu^{2}}\right)^{1 / 3},
$$

where $E_{0}$ is the evaporation factor (default value equal to unity), $\Delta \eta$ the concentration difference at the surface and at distance, $\rho$ the atmospheric density, $\Delta \rho$ the $\mathrm{CO}_{2}$ density difference at the surface and at distance, $D$ the diffusion coefficient of water in $\mathrm{CO}_{2}, g$ the acceleration due to gravity and $\nu$ the kinematic viscosity of $\mathrm{CO}_{2}$ (cf. also Sears and Moore 2005). The term $\Delta \eta$ is given by

$$
\Delta \eta=\frac{\rho_{\mathrm{w}}^{\mathrm{sat}}}{\rho}=\frac{M_{\mathrm{w}} P_{\mathrm{sat}}}{M_{\mathrm{c}} P}
$$

where $\rho_{\mathrm{w}}^{\text {sat }}$ is the saturation density of water vapour at the surface temperature $T$ and $M_{\mathrm{w}}$ and $M_{\mathrm{c}}$ are the molecular weights of water and carbon dioxide, respectively. The terms $\rho$ and $\Delta \rho / \rho$ are calculated by applying the ideal gas law,

$$
\rho=\frac{M_{\mathrm{c}} P}{R T}, \quad \frac{\Delta \rho}{\rho}=\frac{\left(M_{\mathrm{c}}-M_{\mathrm{w}}\right) P_{\mathrm{sat}}}{M_{\mathrm{c}} P-\left(M_{\mathrm{c}}-M_{\mathrm{w}}\right) P_{\mathrm{sat}}},
$$

where $R$ is the universal gas constant. Parameter values are given in Table 1.

\begin{tabular}{ll}
\hline Quantity & Value \\
\hline Gravity acceleration, $g$ & $3.72 \mathrm{~m} \mathrm{~s}^{-2}$ \\
Diffusion coefficient, $D$ & $1.4 \times 10^{-3} \mathrm{~m}^{2} \mathrm{~s}^{-1}$ \\
Kinematic viscosity of $\mathrm{CO}_{2}, \nu$ & $6.93 \times 10^{-4} \mathrm{~m}^{2} \mathrm{~s}^{-1}$ \\
Universal gas constant, $R$ & $8.314 \mathrm{~J} \mathrm{~mol}^{-1} \mathrm{~K}^{-1}$ \\
Molar mass of water, $M_{\mathrm{w}}$ & $1.802 \times 10^{-2} \mathrm{~kg} \mathrm{~mol}^{-1}$ \\
Molar mass of $\mathrm{CO}_{2}, M_{\mathrm{c}}$ & $4.401 \times 10^{-2} \mathrm{~kg} \mathrm{~mol}^{-1}$ \\
\hline
\end{tabular}

Table 1: Physical parameters for the evaporation model of MAIC-2.

Sears and Moore (2005) state that the evaporation rate of ice is probably about half that of liquid water. In addition, any significant evaporation of the dusty ice of the PLDs will lead to an enrichment of dust at the surface, thus producing an insulating layer which hampers further evaporation. These effects can be accounted for by setting the evaporation 


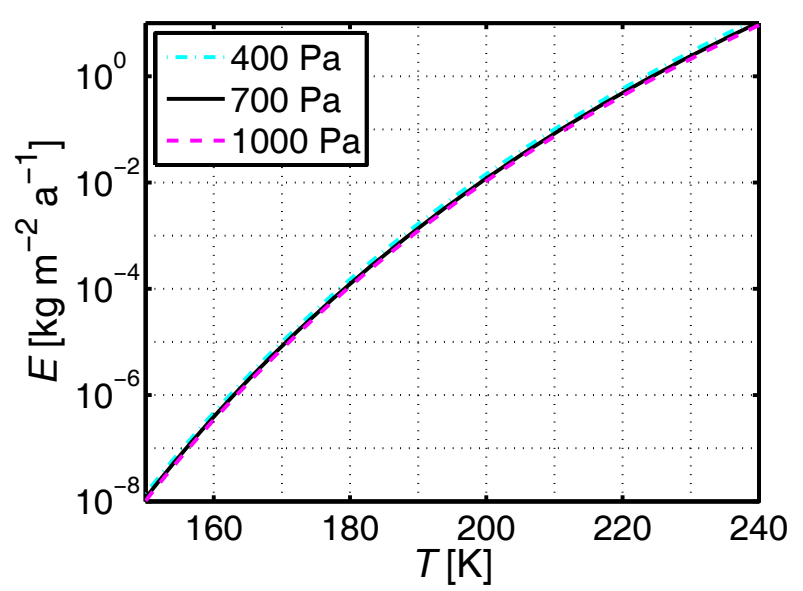

Figure 5: Dependence of the evaporation rate $E$ on the surface temperature $T$ for surface pressures $P=400,700$ and $1000 \mathrm{~Pa}$, and $E_{0}=0.1$.

factor $E_{0}$ in Eq. (12) to a value less than unity, and we will use a standard value of $E_{0}=0.1$ (this choice will be detailed below in Sect. 3.2).

The dependence of evaporation on surface temperature and pressure is illustrated in Fig. 5. The temperature dependence is evidently very strong, while the pressure dependence is weak. Owing to the strong temperature dependence and the short reaction time of evaporation on changing conditions, it is not sufficient to calculate evaporation rates on the basis of daily mean temperatures. Therefore, we parameterise the daily cycle $T_{\mathrm{DC}}$ as follows,

$$
T_{\mathrm{DC}}(\varphi, t)=T(\varphi, t)+\hat{T}(\varphi) \cos \left(\frac{2 \pi t}{1 \mathrm{sol}}\right),
$$

with the amplitude

$$
\hat{T}(\varphi)=\hat{T}_{\mathrm{EQ}}\left[1-\left(\frac{|\varphi|}{90^{\circ}}\right)^{3}\right]
$$

The amplitude at the equator is set to $\hat{T}_{\mathrm{EQ}}=30 \mathrm{~K}$. This choice provides a good agreement with the amplitudes measured by the surface missions Mars Pathfinder $\left(19^{\circ} \mathrm{N}, \hat{\mathrm{T}} \sim 30 \mathrm{~K}\right)$, Viking Lander $1\left(22^{\circ} \mathrm{N}, \hat{T} \sim 30 \mathrm{~K}\right)$ and Viking Lander $2\left(48^{\circ} \mathrm{N}, \hat{T} \sim 25 \mathrm{~K}\right)($ Tillman 2001) as well as the requirement $\hat{T}=0 \mathrm{~K}$ for the poles (Fig. 6).

For high temperatures (e.g., $T_{\mathrm{DC}}>272.8 \mathrm{~K}$ for $P=700 \mathrm{~Pa}$ ), due to the increasing saturation pressure $P_{\text {sat }}$, the term $\Delta \rho / \rho$ of Eq. (14) becomes larger than unity, goes through a singularity and then becomes negative. This means that $\Delta \rho / \rho$ loses its physical meaning. In that case, we correct the problem by resetting $\Delta \rho / \rho$ to unity.

The above equation (12) is valid for an ice cap in contact with the atmosphere. By contrast, for the case of ground ice, we assume that the evaporation rate is reduced with increasing thickness $H_{\text {reg }}$ of the ice-free regolith layer (which separates the atmosphere from the ground ice),

$$
E \rightarrow E \times \exp \left(-\frac{H_{\mathrm{reg}}}{\gamma_{\mathrm{reg}}}\right),
$$

where $\gamma_{\text {reg }}$ is the regolith-insulation coefficient, chosen as $\gamma_{\mathrm{reg}}=0.1 \mathrm{~m}$. 


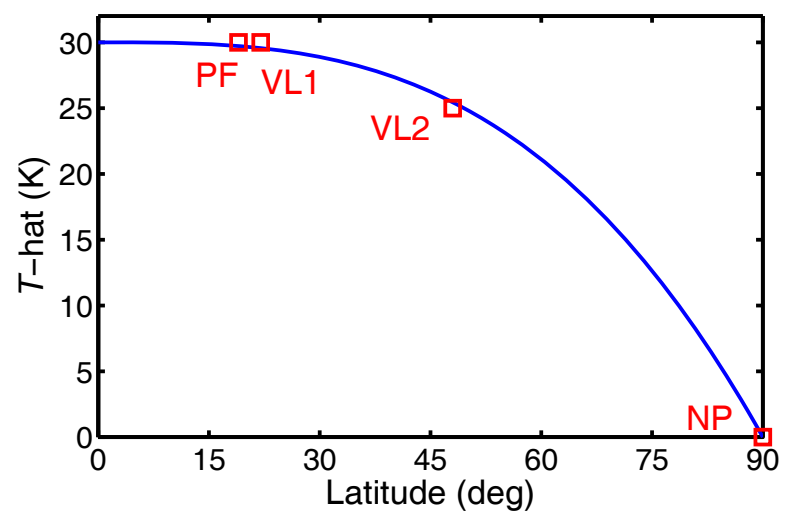

Figure 6: Amplitude $\hat{T}$ of the daily cycle of the surface temperature according to Eq. (16). Comparison with the data for Mars Pathfinder (PF), Viking Lander 1 (VL1) and Viking Lander 2 (VL2) (Tillman 2001) as well as the north pole (NP).

\subsection{Condensation}

The water content $\omega$ in the atmosphere is expressed as an area mass density in units of $\mathrm{kg} \mathrm{m}^{-2}$. Multiplied with the gravity acceleration $g$, this becomes equivalent to the partial pressure of water vapour at the surface. Thus we compare this pressure to the water vapor saturation pressure $P_{\text {sat }}$ and assume that all excess water condenses instantly,

$$
\begin{array}{ll}
\text { If } g \omega>P_{\text {sat }}(T): & \text { excess water }\left[\omega-P_{\text {sat }}(T) / g\right] \\
& \text { determines condensation rate } C, \\
\text { else : } & C=0 .
\end{array}
$$

Note that this is a very simplistic approach because in reality condensation takes place higher in the atmosphere where the temperature may differ considerably from the surface temperature.

\subsection{Transport}

As mentioned above, the water content $\omega$ in the atmosphere is an area mass density. Since the evaporation $E$ (cf. Sect. 2.4) and condensation $C$ (cf. Sect. 2.5) are expressed as mass fluxes in units of $\mathrm{kg} \mathrm{m}^{-2} \mathrm{~s}^{-1}$, its evolution is governed by

$$
\frac{\partial \omega}{\partial t}=-\nabla \cdot \mathbf{F}+E-C
$$

where $\mathbf{F}$ is the horizontal water flux in units of $\mathrm{kg} \mathrm{m}^{-1} \mathrm{~s}^{-1}$.

We approximate the atmospheric water transport by instantaneous mixing (on a time scale of several sols). This can formally be obtained by assuming a diffusive flux,

$$
\mathbf{F}=-K \nabla \omega
$$

with the limit of infinite diffusivity, $K \rightarrow \infty$.

The MAIC version with the LIT scheme of Sect. 2.2 and the surface mass balance of water ice that results from Sects. 2.3-2.6 is referred to as "MAIC-2". 


\section{$2.7 \quad$ Ice evolution}

With the condensation $C$ and the evaporation $E$, the net mass balance $a_{\text {net }}$ of the ice caps, expressed in units of $\mathrm{ms}^{-1}$ ice equivalent, is

$$
a_{\text {net }}=\frac{C-E}{\rho_{\text {ice }}},
$$

where $\rho_{\text {ice }}=910 \mathrm{~kg} \mathrm{~m}^{-3}$ is the density of ice. For a static model (glacial flow neglected), the evolution of the ice thickness, $H$, is then simply

$$
\frac{\partial H}{\partial t}=a_{\text {net }}
$$

Note that we allow for negative ice thicknesses $(H<0)$. Such a situation is interpreted as ground ice under an ice-free regolith layer of thickness $H_{\text {reg }}=|H|$. The thickness of the ground ice layer itself is undefined.

The validity of the assumption of negligible glacial flow is debatable. On the one hand, modelled ice flow speeds on Mars are generally slow, even during periods of high obliquity (Greve et al. 2004, Greve and Mahajan 2005). One the other hand, locally enhanced glacial flow may occur near chasmata and troughs of the PLDs (Hvidberg 2003, Greve 2008), and Winebrenner et al. (2008) argue that the overall topography of Gemina Lingula (also known as Titania Lobe), the lobe of the northern PLDs to the south of Chasma Boreale, was likely shaped by past glacial flow. In this study, we will stick to the simple, static ice model, but consider the inclusion of glacial flow for future work.

\section{Simulations}

We will now discuss the application of MAIC-2 to two different sets of scenarios. The first set is of "academic" nature with orbital parameters kept constant over time, whereas the second one employs a realistic, time-dependent forcing over the last 10 million years. In order to carry out these simulations, a finite-difference/finite-volume discretisation of the model equations of MAIC-2 has been derived (see Appendix A for details) and coded in the Fortran program maic2.F90 (available as free software at http://maic2.greveweb.net). Instantaneous mixing of water vapour in the atmosphere is assumed (diffusivity $K \rightarrow \infty$ ), a time step of $\Delta t=0.02 \mathrm{a}(\approx 7 \mathrm{sols})$ and an equidistant grid spacing of $\Delta \varphi=1^{\circ}$ are chosen (the formulation in Appendix A allows also for non-equidistant grid spacings), and the initial ice distribution is a layer of $19 \mathrm{~m}$ thickness on the entire surface of Mars. The latter value accounts for the ice inventory of the present-day PLDs, $\sim 1.14 \times 10^{6} \mathrm{~km}^{3}$ in the north (Grima et al. 2009) and $\sim 1.6 \times 10^{6} \mathrm{~km}^{3}$ in the south (Plaut et al. 2007).

\subsection{Constant orbital parameters}

Simulations \#1-4 have been run over 10 million years with constant orbital parameters as follows:

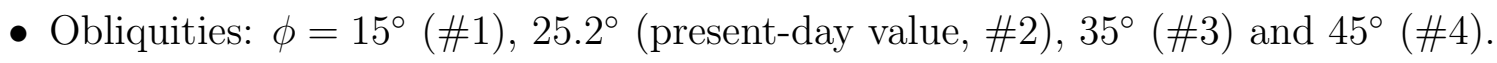


- Eccentricity: $\varepsilon=0.093$ (present-day value, \#1-4).

- Solar longitude of perihelion: $L_{\mathrm{s}, \mathrm{p}}=251.0^{\circ}$ (present-day value, $\# 1-4$ ).

The evaporation factor in Eq. (12) is set to the standard value $E_{0}=0.1$ for all four simulations.

The resulting net mass balance of water ice in the first Martian year of simulation \#2 (all parameters at their present-day values) is shown in Fig. 7. The distribution resembles that of the surface temperature (Fig. 4a). The seasonal $\mathrm{CO}_{2}$ caps are efficient cold traps for atmospheric water vapour, which leads to strongly positive mass balances in the Martian polar regions for most of the year. By contrast, in the lower latitudes negative mass balances prevail, so that the initial ice layer of constant thickness is redistributed towards the poles.

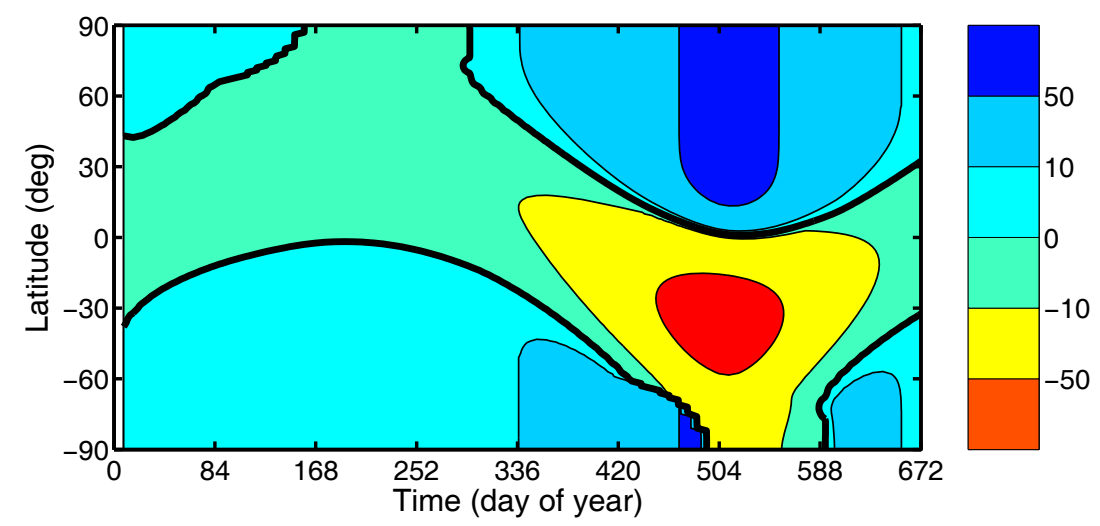

Figure 7: Simulation \#2: Net surface mass balance (in mmice equiv. $\mathrm{a}^{-1}$ ) in the first Martian year. The thick contour shows the equilibrium line (zero mass balance).

The evolution of the ice thickness over the entire simulation time of $10^{7}$ years for all simulations is depicted in Fig. 8. The obliquity of simulation $\# 1\left(\phi=15^{\circ}\right)$ is approximately equal to the minimum value which occurred during the last $4 \mathrm{Ma}$ (Fig. 1). The resulting evolution of the ice thickness is shown in Fig. 8a. The simulation produces bipolar ice deposits, somewhat more pronounced in the north than in the south. After $10^{7}$ years, the simulated polar deposits reach maximum thicknesses of $\sim 500 \mathrm{~m}$, which is about a factor 5 thinner than the observed PLDs at present.

For simulation \#2 (Fig. 8b), it is striking that the redistribution of ice towards the poles is strongly antisymmetric. Until $10^{4}$ years, MAIC-2 produces more pronounced ice deposits in the northern hemisphere and less pronounced ones in the southern hemisphere. At $10^{5}$ years and later, the ice migrates entirely to the northern hemisphere and concentrates around the north pole. In fact, the simulated north polar deposits at $10^{7}$ years resemble the currently existing PLDs in extent and thickness (see below, Fig. 10b), while the simulated south polar region is ice-free.

The reason for this behaviour is the hemispheric asymmetry of the daily mean surface temperature (Fig. 4). As a consequence of the closer proximity of Mars to the Sun during southern summer, the southern summer is distinctly warmer than the northern summer. This leads to large evaporation rates during southern summer and thus a large amount of water vapour in the atmosphere, which is trapped preferably in the winter-cold high 

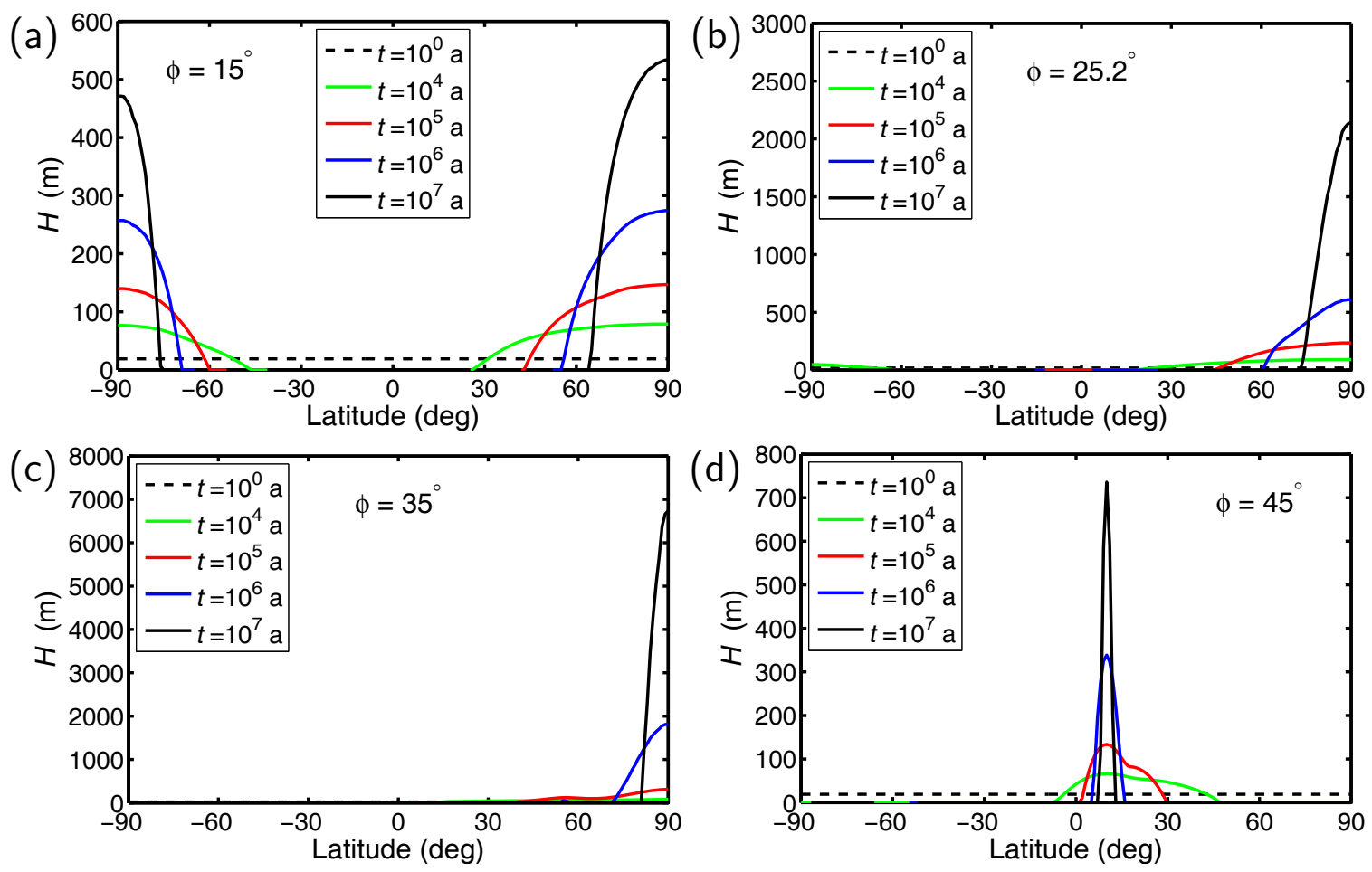

Figure 8: (a) Simulation \#1 $\left(\phi=15^{\circ}\right)$, (b) \#2 $\left(\phi=25.2^{\circ}\right),(\mathrm{c}) \# 3\left(\phi=35^{\circ}\right)$ and $(\mathrm{d}) \# 4$ $\left(\phi=45^{\circ}\right)$ : Evolution of the ice thickness $H$. Note the different scales of the $H$-axes.

northern latitudes. Conversely, the northern summer is less warm, evaporation rates are lower, and therefore the potential for water ice accumulation in the south polar region is much smaller (see also Fig. 7). This holds also for simulation \#1; however, the effect is less pronounced as a result of the weaker seasonal cycle due to the lower obliquity. Hence the hemispheric asymmetry is much weaker for simulation \#1 than for simulation \#2.

For simulation $\# 3$, the obliquity $\left(\phi=35^{\circ}\right)$ is approximately equal to the maximum value during the last $4 \mathrm{Ma}$. Figure $8 \mathrm{c}$ displays the resulting evolution of the ice thickness. The result is similar to that of simulation \#2; however, the concentration of ice around the north pole is more extreme. The simulated north polar deposits at $10^{7}$ years are as thick as $6.7 \mathrm{~km}$, almost 2.5 times thicker than their observed present-day counterparts.

The obliquity of simulation $\# 4\left(\phi=45^{\circ}\right)$ was reached in several maxima during the period of high average obliquity prior to $5 \mathrm{Ma}$ ago. This makes the seasons very extreme. Like in simulations $\# 1-3$, the ice is preferentially deposited in the northern hemisphere (Fig. 8d) due to the warmer southern summers. However, now the poles receive substantial insolation during the respective summer season, so that the northern hemispheric ice deposits are not thickest at the north pole any more. Instead, ice deposition is favoured in the low latitudes, and beyond $10^{4}$ years simulation time the deposits develop a thickness maximum as far south as $\sim 10^{\circ} \mathrm{N}$. 


\subsection{Evolution over the last ten million years}

Simulations \#5-8 attempt at providing realistic, time-dependent scenarios over the last millions of years. To this end, they have been run from $10 \mathrm{Ma}$ ago until today, driven by the history of orbital parameters (obliquity, eccentricity, solar longitude of perihelion) by Laskar et al. (2004). The evaporation factor in Eq. (12) is set to the values $E_{0}=0.05$ (\#5), 0.1 (standard value, \#6), 0.2 (\#7) and 0.3 (\#8).

\begin{tabular}{clccccc}
\hline Sim. & $E_{0}$ & $V_{\text {NPLD }}\left[\mathrm{km}^{3}\right]$ & $V_{\text {SPLD }}\left[\mathrm{km}^{3}\right]$ & $H_{\text {NP }}[\mathrm{m}]$ & $H_{\mathrm{SP}}[\mathrm{m}]$ & \multicolumn{1}{c}{$J$} \\
\hline$\# 5$ & 0.05 & $1.24 \times 10^{6}$ & $1.58 \times 10^{6}$ & 1889 & 2114 & 9.30 \\
$\# 6$ & 0.1 & $1.16 \times 10^{6}$ & $1.65 \times 10^{6}$ & 2404 & 2732 & 2.11 \\
$\# 7$ & 0.2 & $1.02 \times 10^{6}$ & $1.78 \times 10^{6}$ & 2577 & 3170 & 4.96 \\
$\# 8$ & 0.3 & $0.94 \times 10^{6}$ & $1.86 \times 10^{6}$ & 2431 & 3751 & 12.05 \\
\hline Obs. & - & $1.14 \times 10^{6}$ & $1.6 \times 10^{6}$ & 2773 & 2285 & - \\
\hline
\end{tabular}

Table 2: Simulations \#5-8: Evaporation factor $E_{0}$, present-day volume of the north and south polar layered deposits $\left(V_{\mathrm{NPLD}}, V_{\mathrm{SPLD}}\right)$, present-day ice thickness at the north and south pole $\left(H_{\mathrm{NP}}, H_{\mathrm{SP}}\right)$. The last row shows the observed volumes (Plaut et al. 2007, Grima et al. 2009) and ice thicknesses (Zuber et al. 1998, Smith et al. 1999, Greve et al. 2004, see also the caption of Fig. 10). The misfits $J$ have been computed according to Eq. (23).

For the present $(t=0)$, all simulations produce bipolar ice deposits. An overview of the results is given in Table 2 . It shows that, with increasing evaporation factor $E_{0}$, the volume of the northern deposits decreases, while the volume of the southern deposits increases. This holds essentially also for the ice thicknesses at the poles. The only exception is the north polar thickness of simulation \#8 compared to \#7, which decreases by $\sim 6 \%$ even though the ice volume increases by $\sim 4 \%$. These distinctive trends allow to identify the most suitable value of $E_{0}$. To this end, we define the misfit $J$ as follows,

$$
\begin{aligned}
J= & \left(\frac{V_{\mathrm{NPLD}}-V_{\mathrm{NPLD}, \mathrm{obs}}}{\sigma_{V_{\mathrm{NPDL}}}}\right)^{2}+\left(\frac{V_{\mathrm{SPLD}}-V_{\mathrm{SPLD}, \mathrm{obs}}}{\sigma_{V_{\mathrm{SPDL}}}}\right)^{2} \\
& +\left(\frac{H_{\mathrm{NP}}-H_{\mathrm{NP}, \mathrm{obs}}}{\sigma_{H_{\mathrm{NP}}}}\right)^{2}+\left(\frac{H_{\mathrm{SP}}-H_{\mathrm{SP}, \mathrm{obs}}}{\sigma_{H_{\mathrm{SP}}}}\right)^{2} .
\end{aligned}
$$

The standard deviations are introduced to make the various contributions to $J$ commensurate. They are computed from the four respective values of simulations \#5-8,

$$
\begin{aligned}
\sigma_{V_{\mathrm{NPDL}}} & =0.134 \times 10^{6} \mathrm{~km}^{3} \\
\sigma_{V_{\mathrm{SPD}}} & =0.126 \times 10^{6} \mathrm{~km}^{3} \\
\sigma_{H_{\mathrm{NP}}} & =301 \mathrm{~m} \\
\sigma_{H_{\mathrm{SP}}} & =692 \mathrm{~m} .
\end{aligned}
$$

The resulting misfits $J$ are listed in the last column of Table 2. Simulation \#6 with $E_{0}=0.1$ produces clearly the best agreement (and thus $E_{0}=0.1$ is used as standard value throughout this study). The errors of the volumes are as small as $1.6 \%$ for the NPLD and $3.2 \%$ for the SPLD, the ice thickness is $13.3 \%$ too small at the north pole and $19.6 \%$ too 

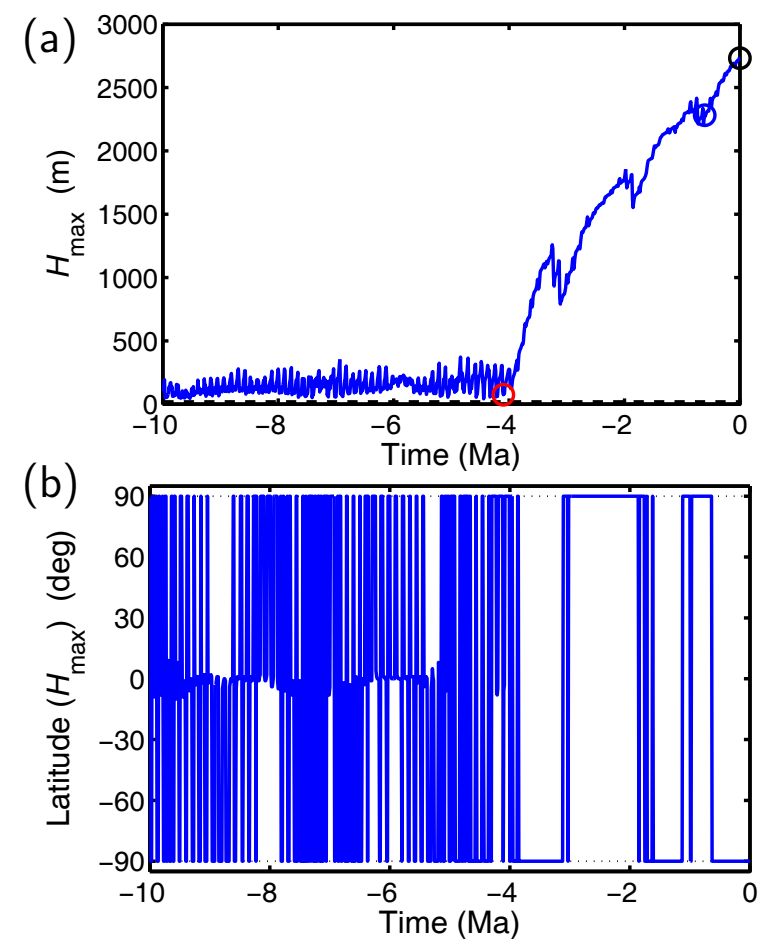

Figure 9: Simulation \#6: (a) Maximum ice thickness (the circle marks correspond to the time slices shown in Fig. 10). (b) Latitude of maximum ice thickness.

large at the south pole. Given the simplicity of the MAIC-2 model, this is a remarkably good overall agreement.

In the following, the best-fit simulation \#6 will be discussed. The maximum ice thickness and its position on the planet are shown in Fig. 9. The simulation produces a mobile glaciation with two distinctly different stages. Stage 1, the period of high average obliquity from 10 until $4 \mathrm{Ma}$ ago, is characterised by ice thicknesses less than $400 \mathrm{~m}$ and rapid changes of the position where the maximum thickness occurs between all latitudes. By contrast, during stage 2 , the period of low average obliquity from $4 \mathrm{Ma}$ ago until today, the position of maximum thickness changes much less rapidly and flip-flops between the poles only ( $47 \%$ of the time at the north pole, $53 \%$ of the time at the south pole). The polar ice deposits grow almost monotonically to their present-day thicknesses, only interrupted by moderate shrinking around $\sim 3.2,1.9$ and $0.7 \mathrm{Ma}$ ago when maximum amplitudes of the main obliquity cycle of 125 ka occurred (see also Fig. 1).

In order to illustrate this behaviour in more detail, Fig. 10 depicts the distribution of the simulated ice thickness for three selected time slices. The first time slice, $4.1 \mathrm{Ma}$ ago (near the end of stage 1$)$, is characterised by a low maximum ice thickness $(74.4 \mathrm{~m})$ which occurs in the low southern latitudes (at $6^{\circ} \mathrm{S}$ ) and a continuous glaciation from the mid southern $\left(47^{\circ} \mathrm{S}\right)$ to the low northern $\left(22^{\circ} \mathrm{N}\right)$ latitudes. In addition, small south polar deposits extend from the pole to $83^{\circ} \mathrm{S}$, whereas the north polar region is entirely ice-free.

The second time slice, $0.61 \mathrm{Ma}$ ago (in stage 2, towards the end of the most recent period of large obliquity amplitudes), shows polar ice deposits only moderately smaller than their present-day counterparts. However, the striking difference compared to the 

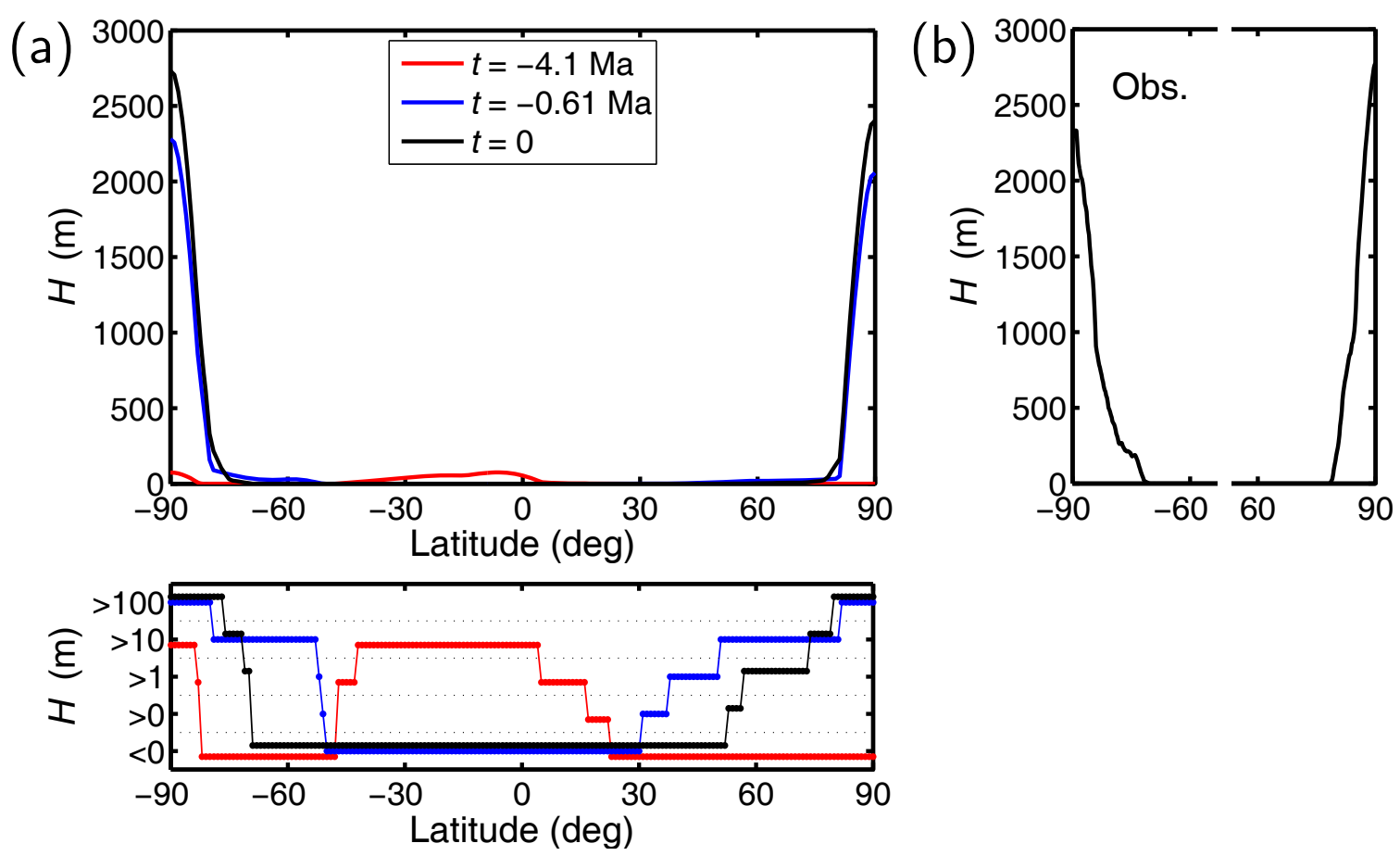

Figure 10: (a) Simulation \#6: Ice thickness $H$ for three selected time slices (which correspond to the marks in Fig. 9a.) The bottom panel shows thickness classes in order to highlight deposits of thin ice not visible in the upper panel. The class " $<0$ " means ground ice (see Sect. 2.7). (b) Observational data of the ice thickness of the present-day PLDs. They have been obtained by subtracting the MOLA surface topography (Zuber et al. 1998, Smith et al. 1999) from the basal topography computed by a smooth extrapolation of the ice-free ground (Greve et al. 2004) and subsequent zonal averaging.

present is the existence of continuous, at least metres-thick ice deposits equatorward to $38^{\circ} \mathrm{N}$ and $52^{\circ} \mathrm{S}$, respectively. These mid-latitude deposits are very mobile; merely $20 \mathrm{ka}$ earlier (0.63 Ma ago), they are entirely absent in the northern hemisphere while extending equatorward to $24^{\circ} \mathrm{S}$ in the southern hemisphere (not shown).

The third time slice is the present $(t=0)$, with an obliquity of $25.2^{\circ}$ following a $\sim 0.3$ Ma period with only small changes (within less than $5^{\circ}$ ). As already discussed above, for the present, the simulation predicts bipolar ice deposits which match the observed volumes within $\sim 3 \%$ and the ice thicknesses at the poles within $\sim 20 \%$. The bulk of the deposits with thicknesses $\geq 100 \mathrm{~m}$ extend to $80^{\circ} \mathrm{N}$ and $77^{\circ} \mathrm{S}$, which also agrees well with the observed values of $80^{\circ} \mathrm{N}$ and $73^{\circ} \mathrm{S}$, respectively. Metres-thick deposits follow equatorward to $57^{\circ} \mathrm{N}$ and $70^{\circ} \mathrm{S}$. This is still compatible with the reality in the south, while it contradicts the reality in the north where such deposits are not observed.

\section{Discussion and conclusion}

The simulations with constant orbital parameters presented in Sect. 3.1 confirm the intuitive idea that low obliquities favour deposition of water ice in high latitudes and vice 
versa. An interesting additional finding is that the polar ice deposits for relatively low obliquities can either occur at one pole only or at both poles, depending on the asymmetry of the seasons in the two hemispheres.

The more realistic best-fit simulation \#6 of Sect. 3.2 with time-dependent orbital forcing from $10 \mathrm{Ma}$ ago until today produces a very good agreement with the observed presentday PLDs. It predicts a mobile glaciation with two distinct stages. During stage 1 , from 10 to $4 \mathrm{Ma}$ ago, ice thicknesses never extend $400 \mathrm{~m}$, and ice is readily exchanged between all latitudes. This exchange is mainly controlled by obliquity, polar deposits being again favoured by relatively low obliquities and lower-latitude deposits by peak obliquities. During stage 2, from $4 \mathrm{Ma}$ ago until today, the north and south polar ice deposits grow essentially monotonically and reach their maximum thicknesses at the present. In particular during the three periods of large obliquity amplitudes around $\sim 3.2,1.9$ and $0.7 \mathrm{Ma}$ ago, the polar deposits continue in thin, very mobile ice deposits which extend far into the mid latitudes at times. The latter result agrees with the findings by Head et al. (2003) who report evidence for "ice ages" on Mars during the period from about 2.1 to $0.4 \mathrm{Ma}$ ago when the obliquity regularly exceeded $30^{\circ}$. According to the authors, the conditions during this period favoured the deposition of metres-thick, dusty, water-ice-rich material down to latitudes of $\sim 30^{\circ}$ in both hemispheres.

A limitation of the results of this study must be noted. The estimated surface ages of the northern (at most $0.1 \mathrm{Ma}$ ) and southern PLDs (about $10 \mathrm{Ma}$ ) by Herkenhoff and Plaut (2000), which are based on crater statistics, are consistent with the simulated growth of ice deposits during the last $4 \mathrm{Ma}$ for the north, but not for the south polar region (see Fig. 10). This may be related to the fact that most of the southern PLDs are covered by dust, whereas the water ice of their northern counterpart is exposed to the atmosphere. Consequently, at least for the present-day situation, the northern PLDs can readily exchange water with the atmosphere, whereas the exchange is blocked to an unknown extent for the southern PLDs. This problem requires further attention. Nevertheless, we conclude that the model MAIC-2 is a very useful tool which, despite its simplicity, can provide substantial insight in the evolution of the Martian surface glaciation over climatological time scales.

\section{Acknowledgements}

The efforts of the scientific editor and reviewers (anonymous) are gratefully acknowledged. This work was partly carried out within the project "Evolution and dynamics of the Martian polar ice caps over climatic cycles" supported by the Research Fund of the Institute of Low Temperature Science, Sapporo, Japan.

\section{A Discrete formulation}

\section{A.1 Numerical grid}

MAIC-2 is a spatially one-dimensional model which features a dependence on latitude only. The grid points are located at monotonically increasing latitudes,

$$
\varphi_{l}, \quad l=0, \ldots, L,
$$


where $\varphi_{0}=-\pi / 2$ (south pole) and $\varphi_{L}=\pi / 2$ (north pole). The generally non-equidistant spacing between subsequent grid points is

$$
\Delta \varphi_{l}=\varphi_{l}-\varphi_{l-1}, \quad l=1, \ldots, L .
$$

Further, we define the latitudes in between grid points (at cell boundaries) as

$$
\varphi_{l \pm 1 / 2}=\frac{\varphi_{l}+\varphi_{l \pm 1}}{2}
$$

Time is discretised uniformly by

$$
t^{n}=t^{0}+n \Delta t, \quad n=0, \ldots, N,
$$

where $t^{0}$ is the initial time, $\Delta t$ the time step and $t^{N}=t^{0}+N \Delta t$ the final time.

\section{A.2 Surface temperature, evaporation, condensation}

Numerical evaluation of the LIT [Eqs. (6)-(10)] and evaporation [Eqs. (12)-(17)] schemes is essentially straightforward and need not be detailed. The daily cycle of the surface temperature [Eq. (15)] for computing evaporation rates is sampled with the sub-daily time step $\Delta t_{\mathrm{DC}}=\frac{1}{8}$ sol. As for condensation, the discrete version of the condition (18) is

$$
C_{l}^{n}=\left\{\begin{array}{cl}
\frac{1}{\Delta t}\left(\omega_{l}^{n}-\frac{P_{\mathrm{sat}}\left(T_{l}^{n}\right)}{g}\right), & \text { if } g \omega_{l}^{n}>P_{\mathrm{sat}}\left(T_{l}^{n}\right), \\
0, & \text { otherwise }
\end{array}\right.
$$

\section{A.3 Instantaneous mixing}

As mentioned in Sect. 2.6, instantaneous mixing of water vapour in the atmosphere can be described as the limit of infinite diffusivity, $K \rightarrow \infty$. A finite-volume discretisation of the diffusion equation with finite diffusivity is described in an earlier version of this paper (archived at arXiv:0903.2688v1 [physics.geo-ph]); however, the limit $K \rightarrow \infty$ cannot be carried out numerically with that scheme. Instead, a two-step procedure has been devised for the case of instantaneous mixing.

In the first step, for any point of the model domain $(l=0, \ldots, L)$, let us compute predictors of the water content at the new time step, $\hat{\omega}_{l}^{n}$, by ignoring the water transport,

$$
\frac{\hat{\omega}_{l}^{n}-\omega_{l}^{n-1}}{\Delta t}=E_{l}^{n}-C_{l}^{n} \text {. }
$$

In the second step, the resulting total amount of water shall be mixed over the entire planet. The total amount of water is obtained by integrating over the grid cells and summing up,

$$
\begin{aligned}
\omega_{\text {tot }}= & 2 \pi R^{2} \hat{\omega}_{0}^{n} \int_{\varphi_{0}}^{\varphi_{1 / 2}} \cos \varphi \mathrm{d} \varphi+\sum_{l=1}^{L-1} 2 \pi R^{2} \hat{\omega}_{l}^{n} \int_{\varphi_{l-1 / 2}}^{\varphi_{l+1 / 2}} \cos \varphi \mathrm{d} \varphi \\
& +2 \pi R^{2} \hat{\omega}_{L}^{n} \int_{\varphi_{L-1 / 2}}^{\varphi_{L}} \cos \varphi \mathrm{d} \varphi \\
= & 2 \pi R^{2}\left\{\hat{\omega}_{0}^{n}\left(1+\sin \varphi_{1 / 2}\right)+\sum_{l=1}^{L-1} \hat{\omega}_{l}^{n}\left(\sin \varphi_{l+1 / 2}-\sin \varphi_{l-1 / 2}\right)\right. \\
& \left.+\hat{\omega}_{L}^{n}\left(1-\sin \varphi_{L-1 / 2}\right)\right\},
\end{aligned}
$$


where $R$ denotes the radius of the planet $(R=3396 \mathrm{~km})$. For all points $l=0, \ldots, L$, the new water content follows by division by the surface of the planet,

$$
\begin{gathered}
\omega_{l}^{n}=\frac{\omega_{\text {tot }}}{4 \pi R^{2}}=\frac{1}{2}\left\{\hat{\omega}_{0}^{n}\left(1+\sin \varphi_{1 / 2}\right)+\sum_{l=1}^{L-1} \hat{\omega}_{l}^{n}\left(\sin \varphi_{l+1 / 2}-\sin \varphi_{l-1 / 2}\right)\right. \\
\left.+\hat{\omega}_{L}^{n}\left(1-\sin \varphi_{L-1 / 2}\right)\right\} .
\end{gathered}
$$

\section{A.4 Ice evolution}

The discretisation of the ice-thickness equation (22) is straightforward. By using an Euler backward step for the time derivative, we obtain

$$
\frac{H_{l}^{n}-H_{l}^{n-1}}{\Delta t}=\left(a_{\text {net }}\right)_{l}^{n}=\frac{C_{l}^{n}-E_{l}^{n}}{\rho_{\text {ice }}} .
$$

\section{References}

Armstrong, J. C., C. B. Leovy and T. Quinn. 2004. A 1 Gyr climate model for Mars: new orbital statistics and the importance of seasonally resolved polar processes. Icarus, 171 (2), 255-271. doi:10.1016/j.icarus.2004.05.007.

Forget, F., F. Hourdin, R. Fournier, C. Hourdin, O. Talagrand, M. Collins, S. R. Lewis, P. L. Read and J.-P. Huot. 1999. Improved general circulation models of the Martian atmosphere from the surface to above $80 \mathrm{~km}$. J. Geophys. Res., 104 (E10), 24155-24175.

Gierasch, P. and R. Goody. 1968. A study of the thermal and dynamical structure of the martian lower atmosphere. Planet. Space Sci., 16 (5), 615-646.

Greve, R. 2008. Scenarios for the formation of Chasma Boreale, Mars. Icarus, 196 (2), 359-367. doi:10.1016/j.icarus.2007.10.020.

Greve, R. and R. A. Mahajan. 2005. Influence of ice rheology and dust content on the dynamics of the north-polar cap of Mars. Icarus, 174 (2), 475-485. doi:10.1016/j.icarus.2004.07.031.

Greve, R., R. A. Mahajan, J. Segschneider and B. Grieger. 2004. Evolution of the north-polar cap of Mars: a modelling study. Planet. Space Sci., 52 (9), 775-787. doi:10.1016/j.pss.2004.03.007.

Grima, C., W. Kofman, J. Mouginot, R. J. Phillips, A. Hérique, D. Biccari, R. Seu and M. Cutigni. 2009. North polar deposits of Mars: Extreme purity of the water ice. Geophys. Res. Lett., 36, L03203. doi:10.1029/2008GL036326.

Haberle, R. M., J. R. Murphy and J. Schaeffer. 2003. Orbital change experiments with a Mars general circulation model. Icarus, 161 (1), 66-89.

Head, J. W., J. F. Mustard, M. A. Kreslavsky, R. E. Milliken and D. R. Marchant. 2003. Recent ice ages on Mars. Nature, 426 (6968), 797-802. doi:10.1038/nature02114.

Herkenhoff, K. E. and J. J. Plaut. 2000. Surface ages and resurfacing rates of the polar layered deposits on Mars. Icarus, 144 (2), 243-253.

Hvidberg, C. S. 2003. Relationship between topography and flow in the north polar cap on Mars. Ann. Glaciol., 37, 363-369. 
Ingersoll, A. P. 1970. Mars: Occurrence of liquid water. Science, 168 (3934), 972-973.

Jakosky, B. M. 1985. The seasonal cycle of water on Mars. Space Sci. Rev., 41, 131-200.

James, P. B., H. H. Kieffer and D. A. Paige. 1992. The seasonal cycle of carbon dioxide on Mars. In: H. H. Kieffer, B. M. Jakosky, C. W. Snyder and M. S. Matthews (Eds.), Mars, pp. 934-968. University of Arizona Press, Tucson, AZ, USA.

Laskar, J., A. C. M. Correia, M. Gastineau, F. Joutel, B. Levrard and P. Robutel. 2004. Long term evolution and chaotic diffusion of the insolation quantities of Mars. Icarus, 170 (2), 343-364. doi:10.1016/j.icarus.2004.04.005.

Lewis, S. R., M. Collins, P. L. Read, F. Forget, F. Hourdin, R. Fournier, C. Hourdin, O. Talagrand and J.-P. Huot. 1999. A climate database for Mars. J. Geophys. Res., 104 (E10), 24177-24194.

Michelangeli, D. V., O. B. Toon, R. M. Haberle and J. B. Pollack. 1993. Numerical simulations of the formation and evolution of water ice clouds in the Martian atmosphere. Icarus, 102 (2), $261-285$.

Murray, F. W. 1967. On the computation of saturation vapor pressure. J. Appl. Meteorol., 6, 203-204.

Plaut, J. J., G. Picardi, A. Safaeinili, A. B. Ivanov, S. M. Milkovich, A. Cicchetti, W. Kofman, J. Mouginot, W. M. Farrell, R. J. Phillips, S. M. Clifford, A. Frigeri, R. Orosei, C. Federico, I. P. Williams, D. A. Gurnett, E. Nielsen, T. Hagfors, E. Heggy, E. R. Stofan, D. Plettemeier, T. R. Watters, C. J. Leuschen and P. Edenhofer. 2007. Subsurface radar sounding of the south polar layered deposits of Mars. Science, 316 (5821), 92-95. doi:10.1126/science.1139672.

Pollack, J. B., R. M. Haberle, J. Schaeffer and H. Lee. 1990. Simulation of the general circulation of the Martian atmosphere 1. Polar processes. J. Geophys. Res., 95 (B2), 1447-1473.

Read, P. L., M. Collins, F. Forget, R. Fournier, F. Hourdin, S. R. Lewis, O. Talagrand, F. W. Taylor and N. P. J. Thomas. 1997. A GCM climate database for Mars: for mission planning and for scientific studies. Adv. Space Res., 19, 1213-1222.

Richardson, M. I. and R. J. Wilson. 2002. A topographically forced asymmetry in the martian circulation and climate. Nature, 416 (6878), 298-301. doi:10.1038/416298a.

Sears, D. W. G. and S. R. Moore. 2005. On laboratory simulation and the evaporation rate of water on Mars. Geophys. Res. Lett., 32 (16), L16202. doi:10.1029/2005GL023443.

Segschneider, J., B. Grieger, H. U. Keller, F. Lunkeit, E. Kirk, K. Fraedrich, A. Rodin and R. Greve. 2005. Response of the intermediate complexity Mars Climate Simulator to different obliquity angles. Planet. Space Sci., 53 (6), 659-670. doi:10.1016/j.pss.2004.10.003.

Smith, D. E., M. T. Zuber, S. C. Solomon, R. J. Phillips, J. W. Head, J. B. Garvin, W. B. Banerdt, D. O. Muhleman, G. H. Pettengill, G. A. Neumann, F. G. Lemoine, J. B. Abshire, O. Aharonson, C. D. Brown, S. A. Hauck, A. B. Ivanov, P. J. McGovern, H. J. Zwally and T. C. Duxbury. 1999. The global topography of Mars and implications for surface evolution. Science, 284 (5419), 1495-1503. 
Stenzel, O. J., B. Grieger, H. U. Keller, R. Greve, K. Fraedrich, E. Kirk and F. Lunkeit. 2007. Coupling Planet Simulator Mars, a general circulation model of the Martian atmosphere, to the ice sheet model SICOPOLIS. Planet. Space Sci., 55 (14), 2087-2096. doi: 10.1016/j.pss.2007.09.001.

Takahashi, Y. O., H. Fujiwara, H. Fukunishi, M. Odaka, Y.-Y. Hayashi and S. Wanabe. 2003. Topographically induced north-south asymmetry of the meridional circulation in the Martian atmosphere. J. Geophys. Res., 108 (E3), 5018. doi:10.1029/2001JE001638.

Tillman, J. E. 2001. Mars: Temperature overview. Online publication. URL http://www-k12.atmos. washington.edu/k12/, retrieved 2009-07-15.

Winebrenner, D. P., M. R. Koutnik, E. D. Waddington, A. V. Pathare, B. C. Murray, S. Byrne and J. L. Bamber. 2008. Evidence for ice flow prior to trough formation in the martian north polar layered deposits. Icarus, 195 (1), 90-105. doi:10.1016/j.icarus.2007.11.030.

Zuber, M. T., D. E. Smith, S. C. Solomon, J. B. Abshire, R. S. Afzal, O. Aharonson, K. Fishbaugh, P. G. Ford, H. V. Frey, J. B. Garvin, J. W. Head, A. B. Ivanov, C. L. Johnson, D. O. Muhleman, G. A. Neumann, G. H. Pettengill, R. J. Phillips, X. Sun, H. J. Zwally, W. B. Banerdt and T. C. Duxbury. 1998. Observations of the north polar region of Mars from the Mars Orbiter Laser Altimeter. Science, 282 (5396), 2053-2060. 